\title{
Methods of measurement signal acquisition from the rotational flow meter for frequency analysis
}

\author{
Dariusz Świsulski ${ }^{1, *}$, Robert Hanus $^{2}$, Marcin Zych $^{3}$, and Leszek Petryka ${ }^{4}$ \\ ${ }^{1}$ Gdańsk University of Technology, Faculty of Electrical and Control Engineering, 80-233 Gdańsk, Poland \\ ${ }^{2}$ Rzeszów University of Technology, Faculty of Electrical and Computer Engineering, 35-959 Rzeszów, Poland \\ ${ }^{3}$ AGH University of Science and Technology, Faculty of Geology, Geophysics and Environmental Protection, 30-059 Kraków, Poland \\ ${ }^{4}$ AGH University of Science and Technology, Faculty of Physics and Applied Computer Science, 30-059 Kraków, Poland
}

\begin{abstract}
One of the simplest and commonly used instruments for measuring the flow of homogeneous substances is the rotational flow meter. The main part of such a device is a rotor (vane or screw) rotating at a speed which is the function of the fluid or gas flow rate. A pulse signal with a frequency proportional to the speed of the rotor is obtained at the sensor output. For measurements in dynamic conditions, a variable interval between pulses prohibits the analysis of the measuring signal. Therefore, the authors of the article developed a method involving the determination of measured values on the basis of the last inter-pulse interval preceding the moment designated by the timing generator. For larger changes of the measured value at a predetermined time, the value can be determined by means of extrapolation of the two adjacent interpulse ranges, assuming a linear change in the flow. The proposed methods allow analysis which requires constant spacing between measurements, allowing for an analysis of the dynamics of changes in the test flow, eg. using a Fourier transform. To present the advantages of these methods simulations of flow measurement were carried out with a DRH-1140 rotor flow meter from the company Kobold.
\end{abstract}

\section{Principles of methods}

One of the simplest and commonly used instruments for measuring the flow of homogeneous substances is the rotational flow meter.

An essential part of rotary flow metres is the rotor rotating at a speed proportional to the fluid flow rate. The rotor may be vane or screw type.

A vane rotor (Fig. 1) has a symmetrical blade. A flow meter is used for measuring low flow rates in pipelines with a diameter of $40 \mathrm{~mm}$.

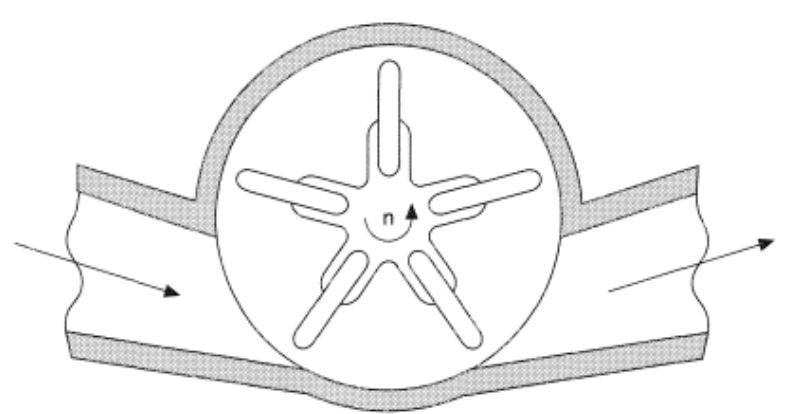

Fig. 1. Turbine flowmeter with rotation normal to line of flow [1].

Screw flow meters (Fig. 2) have a rotor in the shape of a multiple turn screw through which fluid flows axially. Such flow meters may be used for higher flows and diameters.

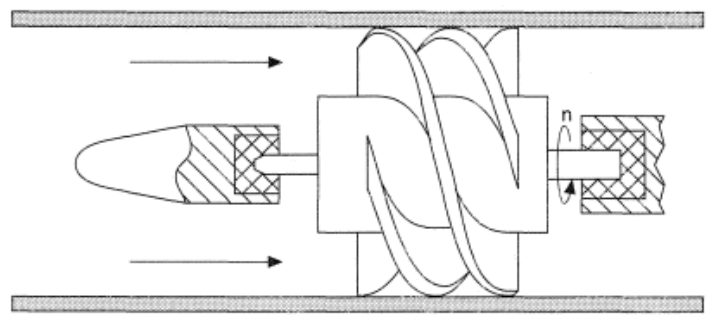

Fig. 2. Turbine flowmeter with rotation in line of flow [1].

The rotational speed of the rotating element is measured for example by an induction sensor. It is proportional to the mean fluid flow rate and its volume. At the output of the inductive sensor we receive a pulse string.

The impulse at the output of the incremental encoder appears at time $t_{i}$, where the average speed at the time of the previous pulse $t_{i-1}$ is equal to the ratio of the angle of rotation of the rotating referred to the inverse of the resolution (sensitivity) $C_{e}$ (number of pulses per revolution) to time $t_{i}-t_{i-1}$, wherein the disk is rotated by this angle, that is, when the condition [2]:

$$
\frac{1}{t_{i}-t_{i-1}} \int_{t_{i-1}}^{t_{i}} \omega(t) d t=\frac{\overline{1}}{t_{i}-t_{i-1}}
$$

where $\omega(t)$ - the value of instantaneous speed.

\footnotetext{
Corresponding author: dariusz.swisulski@pg.gda.pl
} 
The result is a pulse signal $w(t)$ of the distance between the pulse proportional to the volume of the fluid flow (Fig. 3) [3, 4].

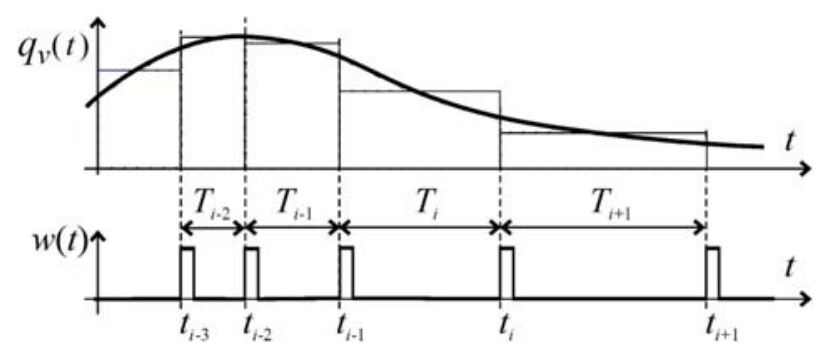

Fig. 3. The pulse signal $w(t)$ of frequency proportional to the flow rate of the fluid flow $q_{v}$.

By measuring the pulse frequency we can calculate the volume flow of the flowing fluid.

\section{The measurements in dynamic conditions}

Traditional methods of digital recording of pulse signals may be divided into two groups: measuring the number of periods of the pulse signal in a predetermined reference time, and measuring the number of pulses of the reference generator during one period or an integer multiple of the pulse signal. For the methods of the second group, the number of signal periods during the measurement of the pulse can be constant or dependent upon their length.

The choice of the appropriate methods is usually dependent on the maximum quantization error. Also, the time taken for the measurement method is dependent of the assumed maximum quantization error. This approach is acceptable when signal frequency is constant in time. The problem arises when during the measurement, the frequency of the signal pulse changes. When measuring flow, when its value changes during the measurement process, this would change the distance between successive pulses. Therefore, the measurements should be used other methods described below.

For measurements in dynamic conditions, a variable interval between pulses does not allow direct frequency analysis of the measurement signal [5].

The methods presented further allow analysis requiring constant spacing between measurements, allowing for an analysis of the dynamics of changes in the test flow, e.g. using a Fourier transform.

Another feature of the measurement systems using such methods is the ability to integrate the channels of voltage, in which the voltage signal is converted to digital form in the analog-digital channels with a frequency signal [6]. Measurements in both types of channels can be sampled using a common timing generator which makes it possible to obtain information about the values measured at the same points in time. This allows e.g. to present the dependence of the measured flow of other physical quantities.

Pulse signal processing methods, depending on the time for which the measurement result is determined can be divided into two groups. In on-line measurements the measured value is determined based on the position of the pulse preceding the moment of measurement. When measuring off-line, first you write to memory the location of all pulses in the time of registration, and after the completion of the measurement determines a value measured in the moments of measurement adopted. Therefore, in this case the measured value is determined not only by the position of the pulse preceding the moment of measurement, but also occurring after that moment.

\section{On-line acquisition}

The easiest way to register a pulse signal $w$ lies in the fact that the value of the signal at any time $t_{k}$ (defined by the pulse from the timing generator, common for analog and frequency channels) is calculated based on the length of the last interval inter-pulse $T_{i}$ preceding the moment $t_{k}$ (Fig. 4).

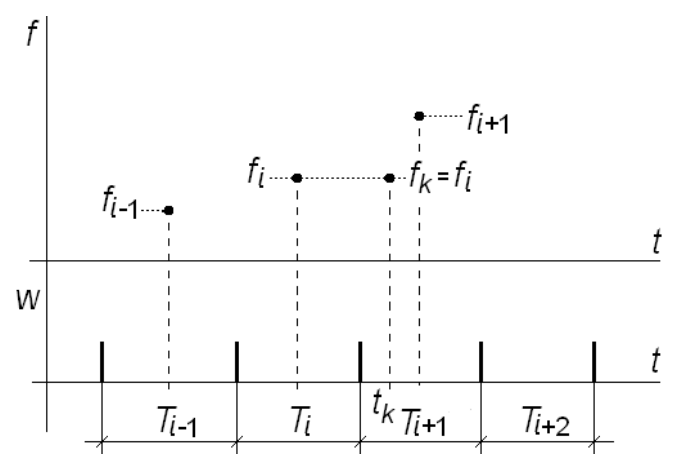

Fig. 4. Measuring the frequency of the last interval between pulses.

If the value of the measured signal changes during the measurement (whereby also the interval between pulses changes) and the longer period of the pulse signal comes to pass (and consequently the longer the time between the end of the period $T_{i}$ and the time $t_{k}$ ) value obtained from the measurement may deviate from that which was at the time of sampling.

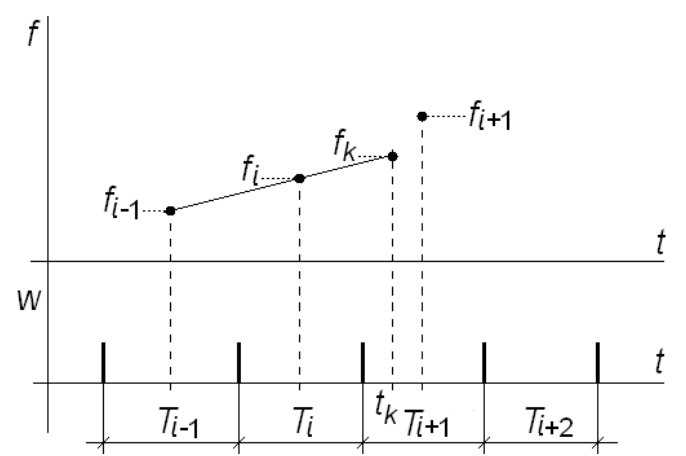

Fig. 5. Frequency measurement with extrapolation of the last two interval periods $T_{i-1}$ and $T_{i}$.

Therefore, in case of change of the measured value, the value at time $t_{k}$ can be calculated from the formula (2) using extrapolation of two adjacent inter-pulse compartments preceding the moment $t_{k}$. A linear change of frequency is assumed and that frequency value $f_{i}$ 
obtained from the measurement interval $T_{i}$ is equal to the instantaneous frequency of the midpoint of this range (Fig. 5).

$$
\begin{array}{r}
f_{k}=f_{i-1}+\frac{\left(f_{i}-f_{i-1}\right)\left(t_{k}-\left(\sum_{j=1}^{i-2} T_{j}+\frac{1}{2} T_{i-1}\right)\right)}{\left(\sum_{j=1}^{i-1} T_{j}+\frac{1}{2} T_{i}\right)-\left(\sum_{j=1}^{i-2} T_{j}+\frac{1}{2} T_{i-1}\right)}= \\
=\frac{1}{T_{i-1}}+\frac{2\left(\frac{1}{T_{i}}-\frac{1}{T_{i-1}}\right)\left(t_{k}-\sum_{j=1}^{i-2} T_{j}-\frac{1}{2} T_{i-1}\right)}{T_{i-1}+T_{i}}
\end{array}
$$

However, such a solution may lead to an increase in measurement error when the frequency does not vary in a manner similar to a linear, but random manner. Frequency determined on the basis of the last two periods may differ significantly from its current value.

\section{Off-line acquisition}

In off-line mode, one must first register the location of pulses, and only after the registration one can determine the measured values in times of sampling. Therefore, to determine the measured value of the pulse signal at any time $t_{k}$ one can use not only the position of the pulse preceding the moment $t_{k}$, but also after they occur. With this solution, instead of using extrapolation, interpolation can also be used.

The simple method implementation in off-line mode is to determine the value measured on the basis of the inter-pulse interval, in which the moment $t_{k}$, i.e. the pulse position before this moment and after that moment. The value of the time $t_{k}$ is calculated from the average value of the frequency between pulses. In the case of linear change in the measured value, the mean value of the time interval $T_{i+1}$ is equal to the instantaneous value in the middle of this range, namely: $f_{k}=f_{i+1}$ (Fig. 6). The method gives satisfactory results when the measured value is of low dynamics.

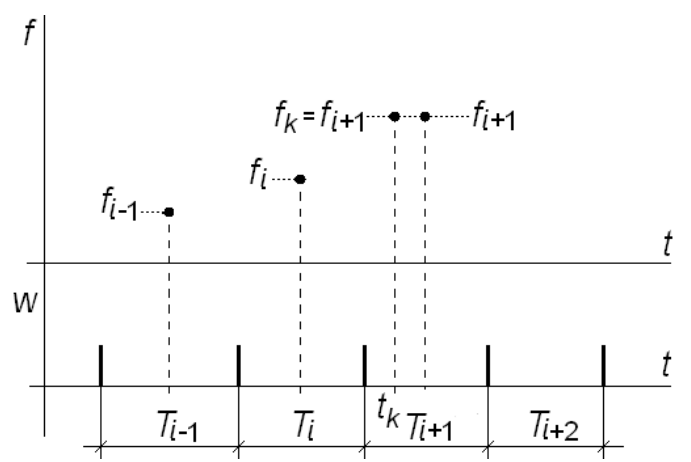

Fig. 6. Measuring the frequency of the current interval between pulses.
If the change of the measured value during the measurement is bigger, the better solution is to determine the frequency of two adjacent inter-pulse periods. One should assume a linear variation of frequencies and that the value of the frequency $f_{i}$ derived from the measurement interval $T_{i}$ is equal to the instantaneous frequency of the midpoint of this range.

Depending on the position of the time $t_{k}$, intervals which determine the frequency $f_{k}$ are $T_{i}$ and $T_{i+1}$ (Fig. 7) or $T_{i+1}$ and $T_{i+2}$ (Fig. 8).

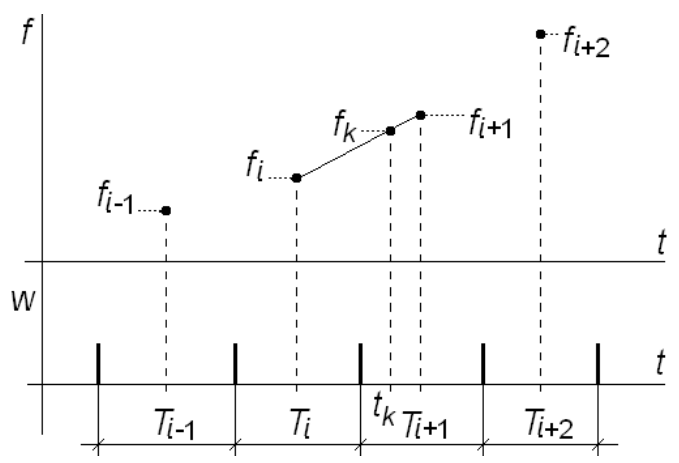

Fig. 7. Frequency measurement based on the length of intervals between two pulses $T_{i}$ and $T_{i+1}$.

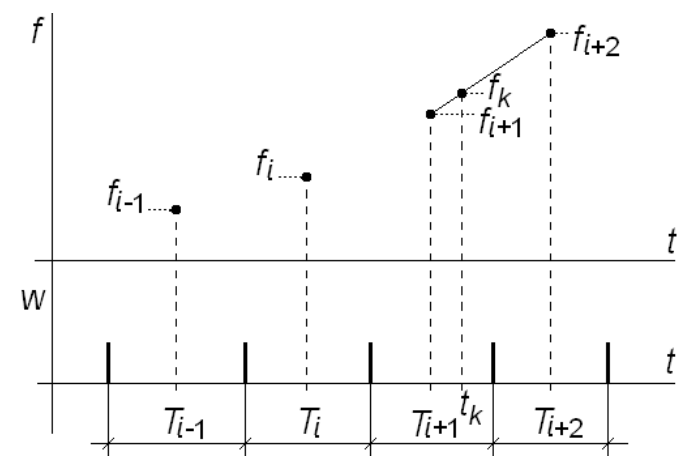

Fig. 8. Frequency measurement based on the length of intervals between two pulses $T_{i+1}$ and $T_{i+2}$.

For the moment $t_{k}$ occurring in the first half of the inter-pulse interval (Fig. 7) the frequency $f_{k}$ is calculated from the formula (3), and for the case where time $t_{k}$ is present in the second half of the interval $T_{i+1}$ (Fig. 8) from equation (4).

$$
\begin{array}{r}
f_{k}=f_{i}+\frac{\left(f_{i+1}-f_{i}\right)\left(t_{k}-\left(\sum_{j=1}^{i-1} T_{j}+\frac{1}{2} T_{i}\right)\right)}{\left(\sum_{j=1}^{i} T_{j}+\frac{1}{2} T_{i+1}\right)-\left(\sum_{j=1}^{i-1} T_{j}+\frac{1}{2} T_{i}\right)}= \\
=f_{i}+\frac{2\left(f_{i+1}-f_{i}\right)\left(t_{k}-\sum_{j=1}^{i-1} T_{j}-\frac{1}{2} T_{i}\right)}{T_{i}+T_{i+1}}
\end{array}
$$




$$
\begin{array}{r}
f_{k}=f_{i+1}+\frac{\left(f_{i+2}-f_{i+1}\right)\left(t_{k}-\left(\sum_{j=1}^{i} T_{j}+\frac{1}{2} T_{i+1}\right)\right)}{\left(\sum_{j=1}^{i+1} T_{j}+\frac{1}{2} T_{i+2}\right)-\left(\sum_{j=1}^{i} T_{j}+\frac{1}{2} T_{i+1}\right)}= \\
=f_{i+1}+\frac{2\left(f_{i+2}-f_{i+1}\right)\left(t_{k}-\sum_{j=1}^{i} T_{j}-\frac{1}{2} T_{i+1}\right)}{T_{i+1}+T_{i+2}}
\end{array}
$$

The sampling rate is determined by the period of time between sampling times $t_{k}$ and $t_{k+1}$.

Simultaneously with the measurement of the frequency at the time $t_{k}$ the voltage channels may be sampled. Such a need occurs when apart from a converter with an output frequency one uses other transducers with voltage output and requires information on the values measured in the same moments of time.

\section{Simulation of recording the signal from the flow meter}

To present the advantages of these methods, simulations of the flow measurements were performed with the DRH-1140 rotor flow meter (Fig. 9) manufactured by Kobold [7].

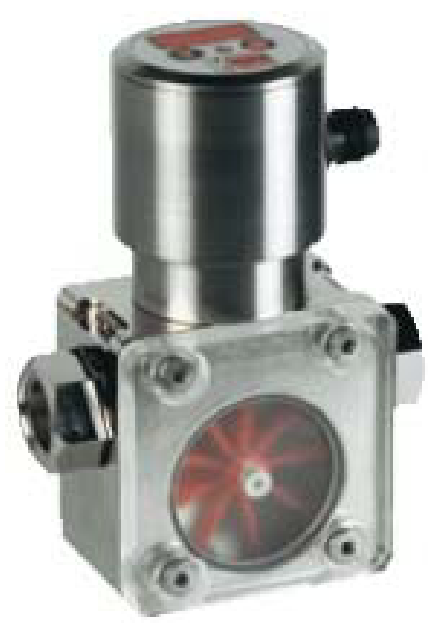

Fig. 9. Rotating Vane Flow Meter DRH-1140 manufactured by Kobold [7].

The range of this flow meter is $261 / \mathrm{min}$, and the frequency of the output frequency signal at a maximum flow rate of $240 \mathrm{~Hz}$. The flow meter uses a fixed geometry impeller blades. Inside the rotor are magnets hermetically isolated from the flowing medium. The Hall effect sensor is located in the housing by the magnetic field magnet, and generates a frequency signal with a frequency proportional to the flow [7].

It was assumed that the flow rate varies sinusoidally in the range of $(20 \pm 4) 1 / \mathrm{min}$, which corresponds to a frequency of the pulse signal at the output of the transducer in the range of $147.7 \mathrm{~Hz}$ to $221.5 \mathrm{~Hz}$.
Figure 10 shows the length of the intervals between successive pulses as a function of their number. Interpulse times vary in the range of $0.00451 \mathrm{~s}$ to 0.00677 seconds.

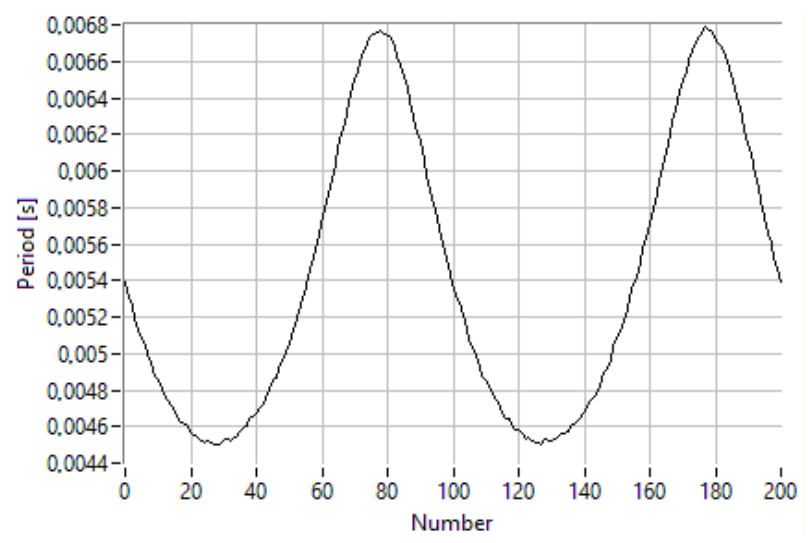

Fig. 10. The length of intervals between pulses as a function of their sequence number.

Due to the varying distances between the moments for which information on the value of the successive inter-pulse intervals was obtained obvious there is fact, that from the string of such obtained values one cannot directly determine the spectrum amplitude. This would result in the appearance of components in the spectrum which do not occur in reality (Fig. 11).

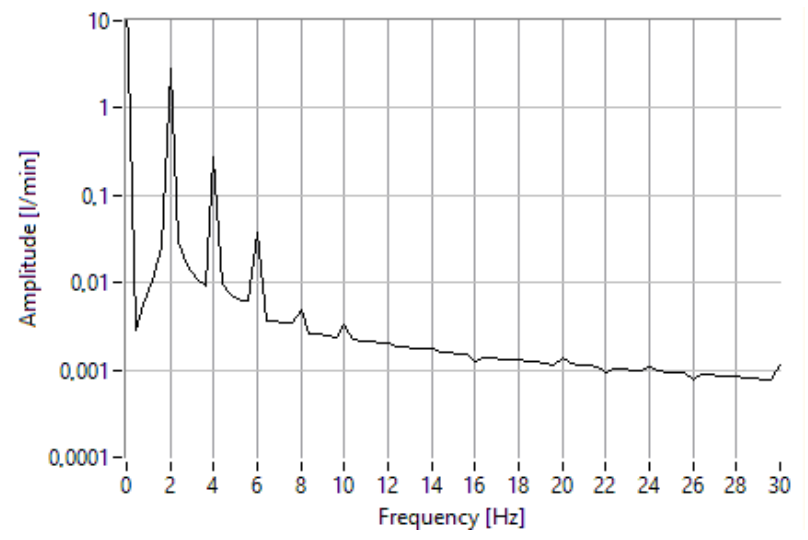

Fig. 11. The spectrum of changes of the measured value determined by the length of intervals between successive pulses.

In order to obtain the spectrum, one can use the previously mentioned methods of acquisition of pulse signal modulated by frequency.

The presented results will be obtained by on-line acquisition of the last inter-pulse interval (as shown in Fig. 4) and the extrapolation of the last two inter-pulse periods (as shown in Fig. 5 and equation (2)).

It was assumed, that the sampling rate is fixed at $0.005 \mathrm{~s}$. The results of the simulation are shown in figures 12 and 13 .

On the basis of these graphs one can reach two conclusions. First, results obtained using the last interpulse interval they are delayed in time relative to the actual changes of the measured value. This follows from the values obtained from the subsequent sampling 
moments based on the length of intervals preceding the moment of inter-pulse sampling. Avoiding this disadvantage is made possible by the use of the interpulse interval, but it is possible for the off-line method (as shown in Fig. 6). This delay does not occur for a method wherein the measured values are determined from extrapolation of the last two inter-pulse periods.

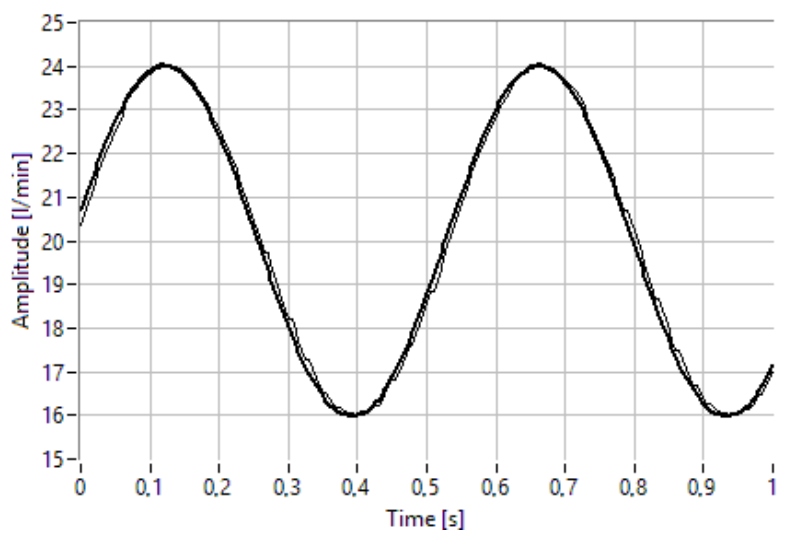

Fig. 12. The course of the measured value as a function of the time appointed for the last inter-pulse interval (thin line) and the extrapolation of the last two inter-pulse periods (bold line).

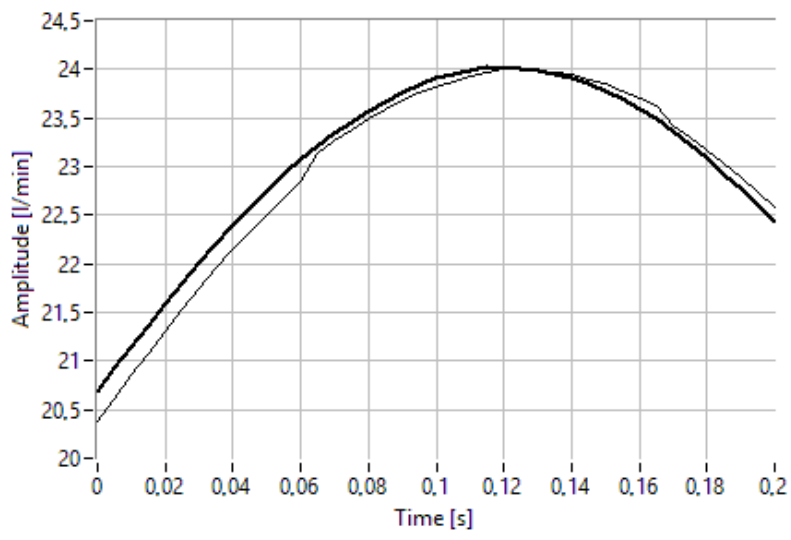

Fig. 13. The enlarged portion of the graph in figure 12 .

The second conclusion that can be drawn from the resuting waveform is a discontinuity in the course of the measured value obtained from the last inter-pulse interval. A discontinuity does not arise in the method in which the mesured values are determined from extrapolation of the last two inter-pulse periods. This is even more evident when one shortens the sampling time. Figure 14 shows the waveforms for the same conditions, as shown in figure 13, but with a reduced sampling time of 0.005 seconds to 0.0005 seconds.

A stepped waveform obtained is due to the fact that the sampling rate is several times smaller than the time of the inter-pulse intervals. Due to this, several successive sampling moments for a given inter-pulse interval give the same value of the measured value determined from the previous inter-pulse interval.

Designated courses of changes of the measured value of the presented methods, thanks to the constant sampling frequency, can be used to determine the amplitude spectrum. This spectrum for both methods is shown in figure 15.

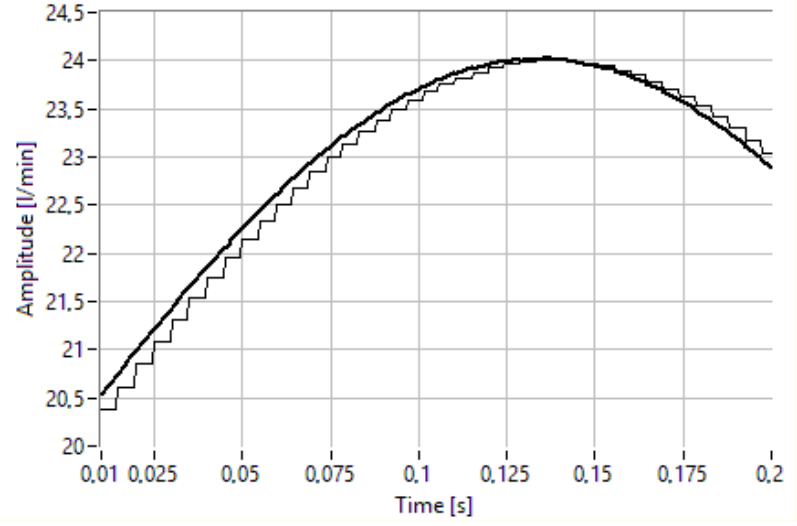

Fig. 14. The course of the measured value as a function of the time appointed for the last inter-pulse interval (thin line) and the extrapolation of the last two inter-pulse periods (bold line) for the sampling period of 0.0005 seconds.

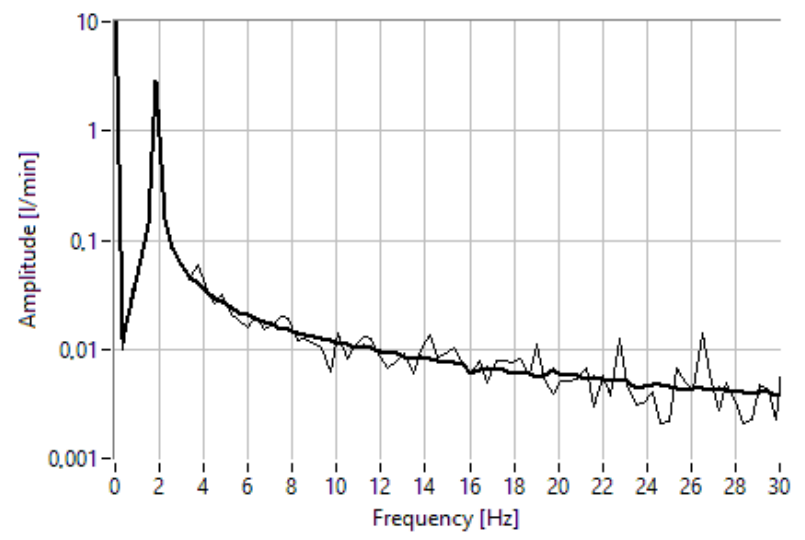

Fig. 15. The amplitude spectrum of the mesured value determined on the basis of the last inter-pulse interval (thin line) and the extrapolation of the last two inter-pulse periods (bold line).

From the graph it can be concluded that the presented methods are well suited for the frequency analysis, although due to the discontinuity of the method discussed earlier, in which one uses single inter-pulse interval, they give inferior results.

The measurement technique should still take into account the interference of the measured value and measurement errors (in this case flow meter errors - the measurement accuracy is $2.5 \%$ of the measuring range given by the manufacturer, and the processing errors of inter-pulse time interval to a digital value). To illustrate the impact of errors and distortions simulated by adding them to the inter-pulse times the Gaussian White Noise with standard deviation of the Gaussian probability density function is equal to $1 \cdot 10^{-5}$. The resulting timedomain waveforms are shown in figures 16 and 17, while in the frequency domain in figure 18.

Comparing the obtained results with the previous ones one can conclude that the errors of measurement and interference have a large impact on final results for the method, in which the measured values are determined from the extrapolation of the last two interpulse periods. This influence is so significant that the results can be even worse than in the case of the method using a single inter-pulse period. 


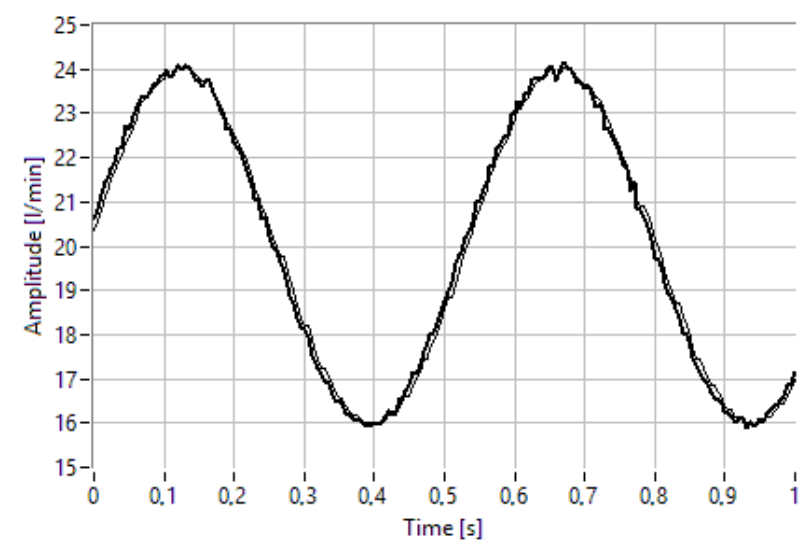

Fig. 16. The course of the measured value as a function of the time appointed for the last inter-pulse interval (thin line) and the extrapolation of the last two inter-pulse periods (bold line) with Gaussian White Noise.

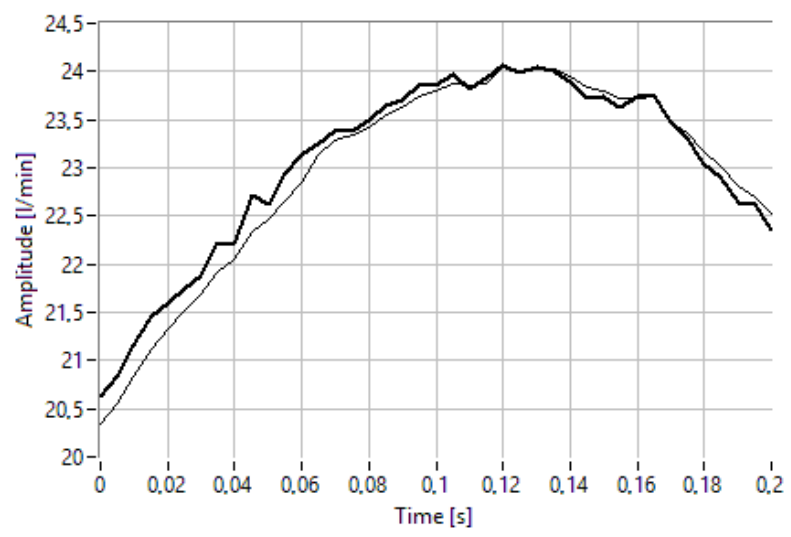

Fig. 17. The enlarged part of the graph in figure 16.

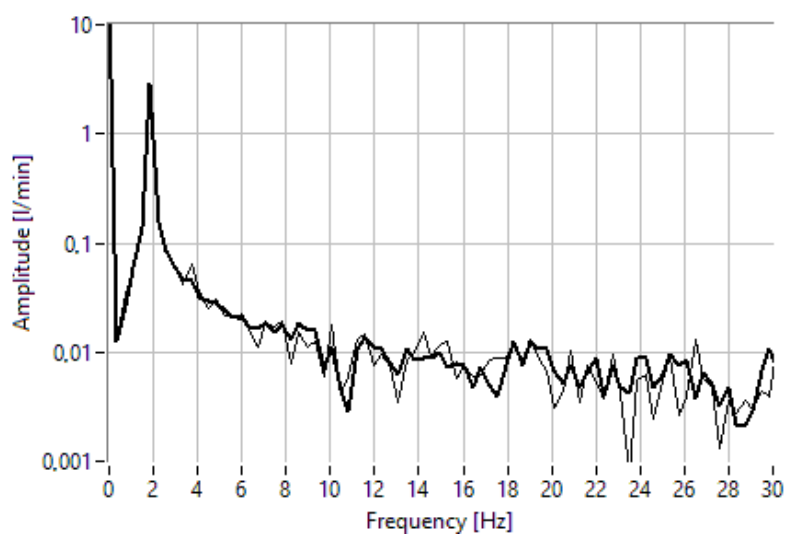

Fig. 18. The amplitude spectrum of the meaured value determined on the basis of the last inter-pulse interval (thin line) and the extrapolation of the last two inter-pulse periods (bold line) with Gaussian White Noise.

\section{Conclusions}

In rotary flow meters the flowrate of the fluid flow is calculated by measuring the frequency of the pulses generated by the incremental encoder.

The presented review developed by the authors of the pulse signal processing methods in certain moments of sampling shows that the choice of method depends on the measurement mode (on-line or off-line) and the nature of the change of the measured value. The simplest solution is to determine the value measured on the basis of a single inter-pulse interval, more complex methods use more periods.

The methods allow to obtain information about the flow rate measured at the same times for the voltage and frequency of the channel, and the samples that were obtained from the frequency channels can be processed by methods requiring a constant sampling frequency (e.g. Fourier Transform).

The results of the flow measurement simulation done with the use of the DRH-1140 rotary flow meter produced by Kobold confirm the advantages of the presented methods.

\section{References}

1. Yu.L. Shekhter, DOI: 10.1615/AtoZ.f.flow_meter ing, http://www.thermopedia.com/content/770/

2. D. Swisulski, Zeszyty Naukowe Wydziału Elektrotechniki i Automatyki Politechniki Gdańskiej 34, 67-70 (2013) (in Polish)

3. N.V. Kirianaki, S.Y. Yurish, N.O. Shpak, V.P. Denega, Data Acquisition and Signal Processing for Smart Sensors (John Wiley \& Sons, New York, 2002)

4. D. Świsulski, E. Pawłowski, Wiadomości Elektrotechniczne 54 (7), 3-9 (2016) (in Polish)

5. E. Pawłowski, Pomiary Automatyka Kontrola 60 (8), 607-609 (2014)

6. D. Świsulski, Przegląd Elektrotechniczny 88 (10b), 29-31 (2012) (in Polish)

7. KOBOLD, Rotating Vane Flow Meter for Liquids, http://fluida-bg.com/assets/files/7817/s4gb_drh.pdf 